\title{
KOSZUL PROPERTY OF DIAGONAL SUBALGEBRAS
}

\author{
NEERAJ KUMAR
}

\begin{abstract}
Let $S=K\left[x_{1}, \ldots, x_{n}\right]$ be a polynomial ring over a field $K$ and $I$ a homogeneous ideal in $S$ generated by a regular sequence $f_{1}, f_{2}, \ldots, f_{k}$ of homogeneous forms of degree $d$. We study a generalization of a result of Conca et al. [9] concerning Koszul property of the diagonal subalgebras associated to $I$. Each such subalgebra has the form $K\left[\left(I^{e}\right)_{e d+c}\right]$, where $c, e \in \mathbf{N}$. For $k=3$, we extend [9, Corollary 6.10] by proving that $K\left[\left(I^{e}\right)_{e d+c}\right]$ is Koszul as soon as $c \geq d / 2$ and $e>0$. We also extend [9, Corollary $6.10]$ in another direction by replacing the polynomial ring with a Koszul ring.
\end{abstract}

1. Introduction. Let $S=K\left[x_{1}, \ldots, x_{n}\right]$ be a polynomial ring over a field $K$ and $I$ a homogeneous ideal in $S$. For large $c$, the algebra $K\left[\left(I^{e}\right)_{c}\right]$ is isomorphic to the coordinate ring of some embedding of the blow up of $\mathbf{P}_{K}^{n-1}$ along the ideal sheaf $\widetilde{I}$ in a projective space [9].

Let Rees $(I)=\bigoplus_{j \geq 0} I^{j} t^{j}$ be the Rees algebra of $I$. Since the polynomial ring $S[t]$ is a bigraded algebra with $S[t]_{(i, j)}=S_{i} t^{j}$, we may consider Rees $(I)$ as a bigraded subalgebra of $S[t]$ with Rees $(I)_{(i, j)}=$ $\left(I^{j}\right)_{i} t^{j}$. Let $c$ and $e$ be positive integers. Let $\triangle=\{(c s, e s): s \in \mathbf{Z}\}$. We call $\triangle$ the $(c, e)$-diagonal of $\mathbf{Z}^{2}[\mathbf{9}]$. Let $R=\oplus_{(i, j) \in \mathbf{Z}^{2}} R_{(i, j)}$ be a bigraded algebra, where $R_{(i, j)}$ denotes the $(i, j)$-th bigraded component of $R$. The (c,e)-diagonal subalgebra of $R_{\delta}$ is defined as the Z-graded algebra $R_{\triangle}=\bigoplus_{s \in \mathbf{Z}} R_{(c s, e s)}$. Similarly, for every bigraded $R$-module $M$, one defines the $(c, e)$-diagonal submodule of $M$ as $M_{\triangle}=$ $\bigoplus_{s \in \mathbf{Z}} M_{(c s, e s)}$. Notice that $M_{\triangle}$ is a module over $R_{\triangle}$.

When $I$ is a homogeneous ideal in $S$ generated by $f_{1}, f_{2}, \ldots, f_{k}$, of homogeneous forms of degree $d$, then Rees $(I)$ is a standard bigraded

2010 AMS Mathematics subject classification. Primary 13D02, Secondary $13 \mathrm{~S} 37$.

Keywords and phrases. Koszul algebra, diagonal subalgebra, Rees algebra, complete intersection.

Received by the editors on September 17, 2012, and in revised form on July 16, 2013. 
algebra by setting $\operatorname{deg} x_{i}=(1,0)$ and $\operatorname{deg} f_{j} t=(0,1)$. We observe that $K\left[\left(I^{e}\right)_{e d+c}\right]$ is the $(c, e)$-diagonal subalgebra of Rees $(I)$. We may also view $K\left[\left(I^{e}\right)_{e d+c}\right]$ as a $K$-subalgebra of $S$ generated by the forms of degree $e d+c$ in the ideal $I^{e}$.

Given a field $K$, a positively graded $K$-algebra $A=\bigoplus_{i \in \mathbf{N}} A_{i}$ with $A_{0}=K$ is Koszul if the field $K$, viewed as an $A$-module via the identification $K=A / A_{+}$, has a linear free resolution. Koszul algebras were introduced by Priddy [15] in 1970. During the last four decades Koszul algebra has been studied in various contexts. A good survey on Koszul algebra was given by Fröberg in [12] during the 90's and recently by Conca, De Negri and Rossi in [10].

Diagonal subalgebras have been intensely studied by several authors (e.g., see [1], [9], [16]) because they naturally appear in Rees algebras and symmetric algebras. In [9], Conca, et al. discuss some algebraic properties of diagonal subalgebras, such as Cohen-Macaulayness and Koszulness. In [14], Kurano et al. showed that Cohen-Macaulayness property holds in [9] even if the polynomial ring is replaced by a CohenMacaulay ring of dimension $d \geq 2$. In this article, we study the Koszul property of certain diagonal subalgebras of bigraded algebras, with focus and applications to diagonals of Rees algebras. We generalize some of the important results of [9] regarding the Koszulness of certain diagonal subalgebras of bigraded algebras.

For any homogeneous ideal $I$, there exist integers $c_{0}, e_{0}$ such that the $K$-algebra $K\left[\left(I^{e}\right)_{e d+c}\right]$ is Koszul for all $c \geq c_{0}$ and $e \geq e_{0}$, see [9, Corollary 6.9]. If $I$ is a complete intersection ideal generated by $f_{1}, f_{2}, \ldots, f_{k}$, of homogeneous forms of degree $d$, then the $K$-algebra $K\left[\left(I^{e}\right)_{e d+c}\right]$ is quadratic if $c \geq d / 2$ and $e>0$; furthermore $K\left[\left(I^{e}\right)_{e d+c}\right]$ is Koszul if $c \geq d(k-1) / k$ and $e>0$, see [9, Corollary 6.10].

The main results of this paper are the following:

(i) Let $I$ be an ideal of the polynomial ring $K\left[x_{1}, \ldots, x_{n}\right]$ generated by a regular sequence $f_{1}, f_{2}, f_{3}$, of homogeneous forms of degree $d$. Then $K\left[\left(I^{e}\right)_{e d+c}\right]$ is Koszul for all $c \geq d / 2$, and $e>0$.

(ii) Let $A$ be a standard graded Koszul ring. Let $I$ be an ideal of $A$ generated by a regular sequence $f_{1}, f_{2}, \ldots, f_{k}$, of homogeneous forms of degree $d$. Then $K\left[\left(I^{e}\right)_{e d+c}\right]$ is Koszul for all $c \geq$ $d(k-1) / k$ and $e>0$. 
Let $R$ be a standard bigraded $K$-algebra. In Section 2 , we study homological properties of shifted modules $R(-a,-b)_{\triangle}$, which play an important role in the transfer of homological information from $R$ to $R_{\triangle}$. It is important to bound the homological invariants of the shifted diagonal module $R(-a,-b)_{\triangle}$ as an $R_{\triangle}$-module. For a bigraded polynomial ring $R$, it is proved in [9] that $R(-a,-b) \triangle$ has a linear $R_{\triangle}$ resolution. Proposition 2.10 is an extension of [9, Theorem 6.2] for certain bigraded complete intersection ideals and crucial in proving Theorem 3.1.

Let $S=K\left[x_{1}, \ldots, x_{n}\right]$ be a polynomial ring. In [9, page 900], the authors mentioned that, for a complete intersection ideal $I$ in $S$ generated by $f_{1}, f_{2}, \ldots, f_{k}$, of homogeneous forms of degree $d$, the algebra $K\left[\left(I^{e}\right)_{e d+c}\right]$ is expected to be Koszul as soon as $c \geq d / 2$. For $k=1,2$, it is obvious. The first nontrivial case is $k=3$. In Section 3, we answer their expectation affirmatively for $k=3$, see Theorem 3.1. The motivation for such generalization came from the work of Caviglia [7] and Caviglia and Conca [8]. Note that, for $k=3$, the result of [8] is just the case: $d=2$ and $c=1$; furthermore, the main result of [7] correspond to the case: $d=2, c=1, f_{1}=x_{1}^{2}, f_{2}=x_{2}^{2}, f_{3}=x_{3}^{2}$ and $n=3$.

In Section 4, we generalize [9, Theorem 6.2] and some of its relevant corollaries. The main result of this section is Theorem 4.5, which is a generalization of [9, Corollary 6.10] regarding the Koszulness of certain diagonals of the Rees algebra of an ideal in the polynomial ring. We show that the Koszulness property holds even if the polynomial ring is replaced by a Koszul ring. The reason for such a generalization comes from the fact that, from a certain point of view, Koszul algebras behave homologically as polynomial rings.

2. Generalities and preliminary results. Let $A$ be a standard graded $K$-algebra, i.e., $A=\bigoplus A_{i}=S / I$, where $S$ is a polynomial ring and $I$ a homogeneous ideal of $S$. For a finitely generated graded $A$-module $M=\oplus M_{i}$, set

$$
t_{i}^{A}(M)=\sup \left\{j: \operatorname{Tor}_{i}^{A}(M, K)_{j} \neq 0\right\},
$$

with $t_{i}^{A}(M)=-\infty$ if $\operatorname{Tor}_{i}^{A}(M, K)=0$. 
Definition 2.1. The Castelnuovo-Mumford regularity $\operatorname{reg}_{A}(M)$ of an $A$-module $M$ is defined to be

$$
\operatorname{reg}_{A} M=\sup \left\{t_{i}^{A}(M)-i: i \geq 0\right\} .
$$

When $A=S$ is a polynomial ring, one has

$$
\operatorname{reg}_{S} M=\max \left\{t_{i}^{S}(M)-i: i \geq 0\right\} .
$$

For a polynomial ring $S=K\left[x_{1}, \ldots, x_{n}\right]$, one can also compute $\operatorname{reg}_{S} M$ via the local cohomology modules $H_{\mathfrak{m}}^{i}(M)$ for $i=0,1, \ldots, n$. One has

$$
\operatorname{reg}_{S} M=\max \left\{j+i: H_{\mathfrak{m}}^{i}(M)_{j} \neq 0\right\} .
$$

Definition 2.2 (Koszul algebra). A standard graded $K$-algebra $A$ is said to be a Koszul algebra if the residue field $K$ has a linear $A$ resolution. Equivalently, $A$ is Koszul when $\operatorname{reg}_{A}(K)=0$.

Example 2.3. Let $A=K[x] /\left(x^{2}\right)$. Then $K$ has a linear $A$-resolution

$$
\cdots \longrightarrow A(-2) \stackrel{\bar{x}}{\rightarrow} A(-1) \stackrel{\bar{x}}{\rightarrow} A \longrightarrow K \longrightarrow 0 .
$$

The property of being a Koszul algebra is preserved under various constructions, in particular under taking tensor products, Segre products and Veronese subrings, see Backelin and Fröberg [4].

Let $A$ be a Koszul algebra, and let $S$ be the polynomial ring mapping onto $A$. Then the regularity of any finitely generated graded module $M$ over $A$ is always finite; in fact, $\operatorname{reg}_{A} M \leq \operatorname{reg}_{S} M$, see Avramov and Eisenbud [3, Theorem 1]. If $M=\bigoplus_{i=a}^{b} M_{i}$ with $M_{b} \neq 0$, then

$$
\operatorname{reg}_{A} M \leq \operatorname{reg}_{S} M=b .
$$

If $A=\bigoplus_{i \geq 0} A_{i}$ is a graded algebra, then the $c$-th Veronese subalgebra is $A^{(c)}=\bigoplus_{i \geq 0} A_{i c}$. An element in $A_{i c}$ is considered to have degree $i$.

Definition 2.4. Consider a standard graded $K$-algebra $A$. Given $k, m \in \mathbf{N}$ and $0 \leq k<m$, we set

$$
V_{A}(m, k)=\bigoplus_{i \in \mathbf{N}} A_{i m+k}
$$


We observe that $A^{(m)}=V_{A}(m, 0)$ is the usual $m$-th Veronese subring of $A$, and that the $V_{A}(m, k)$ are $A^{(m)}$-modules known as the Veronese modules of $A$. For a finitely generated graded $A$-module $M$, similarly we define

$$
M^{(m)}=\bigoplus_{i \in \mathbf{Z}} M_{i m}
$$

We consider $A^{(m)}$ as a standard graded $K$-algebra with homogeneous component of degree $i$ equal to $A_{i m}$ and $M^{(m)}$ as a graded $A^{(m)}$-module with homogeneous components $M_{i m}$ of degree $i$.

Definition 2.5. Let $A$ and $B$ be a positively graded $K$-algebra. Denote by $A \otimes B$ the Segre product

$$
A \underline{\otimes} B=\bigoplus_{i \in \mathbf{N}} A_{i} \otimes_{K} B_{i}
$$

of $A$ and $B$. Given graded modules $M$ and $N$ over $A$ and $B$, one may form the Segre product

$$
M \underline{\otimes} N=\bigoplus_{i \in \mathbf{Z}} M_{i} \otimes_{K} N_{i}
$$

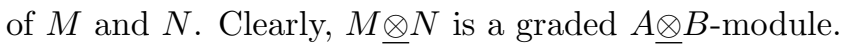

A beautiful introduction to construction of multigraded objects including Segre products is given by Goto and Watanabe in [13, Chapter 4]. Segre products have been studied in the sense of Koszulness by several authors, e.g., Backelin et al. [4], Eisenbud et al. [11], Conca et al. [9], Fröberg [12] and Blum [5]. We will use their results at several occasions in this paper.

Let $A$ and $B$ be Koszul $K$-algebras. Let $M$ be a finitely generated graded $A$-module and $N$ a finitely generated graded $B$-module. Assume $M$ and $N$ have linear resolutions over $A$ and $B$, respectively. Also assume $M \otimes N \neq 0$. Then, by [9, Lemma 6.5], $M \otimes N$ has a linear $A \underline{\otimes} B$-resolution and

$$
\operatorname{reg}_{A \underline{\otimes} B} M \underline{\otimes} N=\max \left\{\operatorname{reg}_{A} M, \operatorname{reg}_{B} N\right\} .
$$

We will use this relation on regularity for Segre products in the proof of Theorems 4.1 and 4.5. 
Remark 2.6. It is to be noticed that [9, Lemma 6.5] also needs the hypothesis $M \underline{\otimes} N \neq 0$. For instance, Assume $M=K$ and $N=K(-1)$. Then $M \underline{\otimes} N=0$, and this leads to inconsistency in (2).

The following lemma is very useful. We will use this lemma in the proof of Proposition 2.10, Theorem 3.1 and Theorem 4.5.

Lemma 2.7 (Technical lemma). Let

$$
\mathbf{M}: \cdots \longrightarrow M_{k+1} \longrightarrow M_{k} \longrightarrow M_{k-1} \longrightarrow \cdots \longrightarrow M_{1} \rightarrow M_{0} \longrightarrow 0,
$$

be a complex of graded $A$-modules with maps of degree 0 . Set $H_{i}=$ $H_{i}(\mathbf{M})$. Then, for every $i \geq 0$, one has

$$
t_{i}^{A}\left(H_{0}\right) \leq \max \{\alpha, \beta\},
$$

where $\alpha=\sup \left\{t_{i-j}^{A}\left(M_{j}\right): j=0, \ldots, i\right\}$ and $\beta=\sup \left\{t_{i-j-1}^{A}\left(H_{j}\right): j=\right.$ $1, \ldots, i-1\}$. Moreover, one has

$$
\operatorname{reg}_{A}\left(H_{0}\right) \leq \max \left\{\alpha^{\prime}, \beta^{\prime}\right\},
$$

where $\alpha^{\prime}=\sup \left\{\operatorname{reg}_{A}\left(M_{j}\right)-j: j \geq 0\right\}$ and $\beta^{\prime}=\sup \left\{\operatorname{reg}_{A}\left(H_{j}\right)-(j+1)\right.$ : $j \geq 1\}$.

Proof. Let $Z_{i}, B_{i}$ and $H_{i}$ denote the $i$ th cycles, boundaries and homology modules respectively. We have short exact sequences

$$
0 \longrightarrow B_{i} \longrightarrow Z_{i} \longrightarrow H_{i} \longrightarrow 0,
$$

and

$$
0 \longrightarrow Z_{i+1} \longrightarrow M_{i+1} \longrightarrow B_{i} \longrightarrow 0,
$$

with $Z_{0}=M_{0}$. Therefore, by [6, Lemma 2.2(a)], one has

$$
\begin{aligned}
t_{i}^{A}\left(H_{0}\right) & \leq \max \left\{t_{i}^{A}\left(M_{0}\right), t_{i-1}^{A}\left(B_{0}\right)\right\}, \\
t_{i-1}^{A}\left(B_{0}\right) & \leq \max \left\{t_{i-1}^{A}\left(M_{1}\right), t_{i-2}^{A}\left(Z_{1}\right)\right\}, \\
t_{i-2}^{A}\left(Z_{1}\right) & \leq \max \left\{t_{i-2}^{A}\left(B_{1}\right), t_{i-2}^{A}\left(H_{1}\right)\right\}, \\
t_{i-2}^{A}\left(B_{1}\right) & \leq \max \left\{t_{i-2}^{A}\left(M_{2}\right), t_{i-3}^{A}\left(Z_{2}\right)\right\},
\end{aligned}
$$


and so on. Summarizing the details, one has:

$$
\begin{aligned}
& t_{i}^{A}\left(H_{0}\right) \\
\leq & \max \left\{t_{i}^{A}\left(M_{0}\right), t_{i-1}^{A}\left(M_{1}\right), \ldots, t_{i-j}^{A}\left(M_{j}\right), t_{i-2}^{A}\left(H_{1}\right), \ldots, t_{i-j-1}^{A}\left(H_{j}\right)\right\} .
\end{aligned}
$$

Take $\alpha=\sup \left\{t_{i-j}^{A}\left(M_{j}\right): j=0, \ldots, i\right\}$ and $\beta=\sup \left\{t_{i-j-1}^{A}\left(H_{j}\right): j=\right.$ $1, \ldots, i-1\}$. Then we obtain the desired result $(3)$ for $t_{i}^{A}\left(H_{0}\right)$. The second inequality (4) follows from ( 3 ).

Let $R=\oplus_{(i, j) \in \mathbf{Z}^{2}} R_{(i, j)}$ be a bigraded standard $K$-algebra. Here standard means that $R_{(0,0)}=K$ and $R$ is generated as a $K$-algebra by the $K$-vector spaces $R_{(1,0)}$ and $R_{(0,1)}$ of finite dimension.

Definition 2.8 (Diagonal subalgebra). Let $R$ be a bigraded standard $K$-algebra. Let $c$ and $e$ be positive integers. Let $\triangle$ be the $(c, e)$-diagonal of $\mathbf{Z}^{2}$. The $(c, e)$-diagonal subalgebra $R_{\triangle}$ of $R$ is defined as

$$
R_{\triangle}=\bigoplus_{s \in \mathbf{Z}} R_{(c s, e s)}
$$

We observe that $R_{\triangle}$ is the $K$-subalgebra of $R$ generated by $R_{(c, e)}$, and hence it is a standard graded $K$-algebra. Similarly, one defines the $(c, e)$-diagonal submodule of any bigraded $R$-module $M=$ $\oplus_{(i, j) \in \mathbf{Z}^{2}} M_{(i, j)}$ as $M_{\triangle}=\bigoplus_{s \in \mathbf{Z}} M_{(c s, e s)}$. Notice that $M_{\triangle}$ is a module over $R_{\triangle}$. The map $M \mapsto M_{\triangle}$, being a selection of homogeneous components, defines an exact functor from the category of bigraded $R$ modules and maps of degree 0 to the category of graded $R_{\triangle}$-modules with maps of degree 0 .

Notation 2.9. We have $\triangle=\{(c s, e s): s \in \mathbf{Z}\}$, but the bounds on $c$ and $e$ change from time to time. Note that $c, s, \triangle$ will always be used in this way, with $c$ and $s$ changing as described. For a real number $\alpha$, we use $\lceil\alpha\rceil$ for the smallest integer $m$ such that $m \geq \alpha$.

For $(a, b) \in \mathbf{Z}^{2}$, let $R(-a,-b)$ be a shifted copy of $R$. By definition,

$$
R(-a,-b)_{\triangle}=\bigoplus_{s \in \mathbf{Z}} R_{(-a+c s,-b+e s)} .
$$


Since $R$ is positively graded, we may consider only those $s \in \mathbf{Z}$ for which $-a+c s \geq 0$ and $-b+e s \geq 0$. Assume $\max \{\lceil a / c\rceil,\lceil b / e\rceil\}=\lceil a / c\rceil$. Then

$$
R(-a,-b)_{\triangle}=\bigoplus_{s \geq\lceil a / c\rceil} R_{(-a+c s,-b+e s)} .
$$

Therefore, $R(-a,-b)_{\triangle}$ is an $R_{\triangle}$-submodule of $R$ generated by

$$
R_{(-a+c\lceil a / c\rceil,-b+e\lceil a / c\rceil)} .
$$

The other case is similar; summarizing the details, one has:

$$
R(-a,-b)_{\triangle}= \begin{cases}R\left(-a+c\left\lceil\frac{a}{c}\right\rceil,-b+e\left\lceil\frac{a}{c}\right\rceil\right) \Delta\left(-\left\lceil\frac{a}{c}\right\rceil\right) & \text { if }\left\lceil\frac{a}{c}\right\rceil \geq\left\lceil\frac{b}{e}\right\rceil ; \\ R\left(-a+c\left\lceil\frac{b}{e}\right\rceil,-b+e\left\lceil\frac{b}{e}\right\rceil\right) \Delta\left(-\left\lceil\frac{b}{e}\right\rceil\right) & \text { if }\left\lceil\frac{a}{c}\right\rceil \leq\left\lceil\frac{b}{e}\right\rceil .\end{cases}
$$

The homological properties of the shifted diagonal module $R(-a,-b)_{\triangle}$ play an important role in the transfer of homological information from $R$ to $R_{\triangle}$. In the following proposition, we try to bound the homological invariant (regularity) of the shifted diagonal module $R(-a,-b)_{\triangle}$ as an $R_{\triangle}$-module. The following proposition is crucial in proving Theorem 3.1.

Proposition 2.10. Let $S=K\left[x_{1}, \ldots, x_{m}, t_{1}, \ldots, t_{n}\right]$ be a polynomial ring bigraded by $\operatorname{deg} x_{i}=(1,0)$ for $i=1, \ldots, m$ and $\operatorname{deg} t_{i}=(0,1)$ for $i=1, \ldots, n$. Let $I$ be an ideal of $S$ generated by a regular sequence with elements all of bidegree $(d, 1)$ and $R=S / I$. Let $d / 2 \leq c<2 d / 3$ and $e>0$. Then:

(a) $R_{\triangle}$ is Koszul.

(b) $\operatorname{reg}_{R_{\triangle}} R(-a,-b)_{\triangle} \leq \max \{\lceil a / c\rceil,\lceil b / e\rceil\}$.

Proof. Let $h$ be the codimension of $I$. The proof is by induction on $h$. If $h=0$, then $R_{\triangle}$ is the Segre product of $K\left[x_{1}, \ldots, x_{m}\right]^{(c)}$ and $K\left[t_{1}, \ldots, t_{n}\right]^{(e)}$. Thus, $R_{\triangle}$ is Koszul by [4]. For (b), see [9, proof of Theorem 6.2].

Assume $h>0$. We may write $R=T /(f)$ where $f$ is a $T$-regular element of bidegree $(d, 1)$ and where $T$ is defined as the quotient of $S$ by an $S$-regular sequence of length $h-1$ of elements of bidegree $(d, 1)$. We have a short exact sequence of $T$-modules:

$$
0 \longrightarrow T(-d,-1) \longrightarrow T \longrightarrow R \longrightarrow 0
$$


and, applying $-\triangle$, we have an exact sequence of $T_{\triangle}$-modules:

$$
0 \longrightarrow T(-d,-1)_{\triangle} \longrightarrow T_{\triangle} \longrightarrow R_{\triangle} \longrightarrow 0 .
$$

By induction, we know $T_{\triangle}$ is Koszul and that $\operatorname{reg}_{T_{\triangle}} T(-d,-1)_{\triangle} \leq$ $\lceil d / c\rceil$. As $d / c \leq 2$, one has

$$
\operatorname{reg}_{T_{\triangle}} R_{\triangle} \leq 1
$$

By [8, Lemma $2.1(3)$, we may conclude that $R_{\triangle}$ is Koszul, as $T_{\triangle}$ is Koszul by induction. Now, to prove (b), we consider the following two cases.

Case 1. Assume $\lceil a / c\rceil<\lceil b / e\rceil$. Shifting $(5)$ by $(-a,-b)$ and then applying $-\triangle$, we get a short exact sequence of $T_{\triangle}$-modules:

$$
0 \longrightarrow T(-a-d,-b-1)_{\triangle} \longrightarrow T(-a,-b)_{\triangle} \longrightarrow R(-a,-b) \triangle \longrightarrow 0 .
$$

So we have:

$$
\begin{aligned}
& \operatorname{reg}_{T_{\triangle}} R(-a,-b)_{\triangle} \\
& \quad \leq \max \left\{\operatorname{reg}_{T_{\triangle}} T(-a,-b)_{\triangle}, \operatorname{reg}_{T_{\triangle}} T(-a-d,-b-1) \triangle-1\right\} .
\end{aligned}
$$

By induction, one has $\operatorname{reg}_{T_{\triangle}} T(-a,-b)_{\triangle} \leq\lceil b / e\rceil$, and

$$
\operatorname{reg}_{T_{\triangle}} T(-a-d,-b-1) \triangle \leq \max \left\{\left\lceil\frac{a+d}{c}\right\rceil,\left\lceil\frac{b+1}{e}\right\rceil\right\} .
$$

Since

$$
\left\lceil\frac{a+d}{c}\right\rceil \leq\left\lceil\frac{a}{c}\right\rceil+\left\lceil\frac{d}{c}\right\rceil \leq\left(\left\lceil\frac{b}{e}\right\rceil-1\right)+2=\left\lceil\left\lceil\frac{b}{e}\right\rceil+1\right.
$$

and

$$
\left\lceil\frac{b+1}{e}\right\rceil \leq\left\lceil\frac{b}{e}\right\rceil+1
$$

we conclude that $\operatorname{reg}_{T_{\triangle}} T(-a-d,-b-1) \triangle \leq\lceil b / e\rceil+1$. Thus, we have

$$
\operatorname{reg}_{T_{\triangle}} R(-a,-b) \triangle \leq\left\lceil\frac{b}{e}\right\rceil .
$$

Since we have already shown that $\operatorname{reg}_{T_{\triangle}} R_{\triangle} \leq 1$, we may conclude by [8, Lemma 2.1 (1)] that

$$
\operatorname{reg}_{R_{\triangle}} R(-a,-b) \triangle \leq\left\lceil\frac{b}{e}\right\rceil .
$$


Case 2. Assume $\lceil a / c\rceil \geq\lceil b / e\rceil$. Setting $P=\left(t_{1}, \ldots, t_{n}\right) \subset S$, we have

$$
R(-a,-b)_{\triangle}=R\left(-a+c\left\lceil\frac{a}{c}\right\rceil,-b+e\left\lceil\frac{a}{c}\right\rceil\right)_{\triangle}\left(-\left\lceil\frac{a}{c}\right\rceil\right) .
$$

So we have to prove that $\operatorname{reg}_{R_{\triangle}} R(\alpha, \beta) \triangle \leq 0$ where $\alpha=-a+c\lceil a / c\rceil$ and $\beta=-b+e\lceil a / c\rceil$. Consider the minimal free (bigraded) resolution of $S / P^{\beta}$ as an $S$-module:

$$
\mathbf{F}: \quad 0 \longrightarrow F_{n} \longrightarrow F_{n-1} \longrightarrow \cdots \longrightarrow F_{1} \longrightarrow F_{0} \longrightarrow 0,
$$

with $F_{0}=S$ and $F_{i}=S^{\sharp}(0,-\beta-i+1)$ for $i>0$ where $\sharp$ denotes some integer depending on $n, \beta$ and $i$ that is irrelevant in our discussion. The homology of $\mathbf{F} \otimes R$ is $\operatorname{Tor}_{\bullet}^{S}\left(S / P^{\beta}, R\right)$. We may as well compute $\operatorname{Tor}_{\bullet}^{S}\left(S / P^{\beta}, R\right)$ as the homology of $S / P^{\beta} \otimes \mathbf{G}$ where $\mathbf{G}$ is a free resolution of $R$ as an $S$-module. By assumption, we may take $\mathbf{G}$ to be a Koszul complex on a sequence of elements of bidegree $(d, 1)$. It follows that:

$$
H_{i}(\mathbf{F} \otimes R)= \begin{cases}\text { a subquotient of }\left(S / P^{\beta}\right)^{\sharp}(-d i,-i) & \text { if } 0 \leq i \leq h ; \\ 0 & \text { if } i>h .\end{cases}
$$

Shifting with $(\alpha, \beta)$ and applying $-\triangle$, we have a complex $(\mathbf{F} \otimes R(\alpha, \beta)) \triangle$. We claim this complex has no homology. Shifting and applying $-\triangle$ are compatible operations with taking homology. Therefore, to prove $(\mathbf{F} \otimes R(\alpha, \beta))_{\triangle}$ has no homology at all, we only need to check that

$$
\left[\left(S / P^{\beta}\right)(-d i+\alpha,-i+\beta)\right]_{\triangle}=0 \text { for all } i .
$$

To prove (6), take the $j$ th degree component

$$
\left[\left[\left(S / P^{\beta}\right)(-d i+\alpha,-i+\beta)\right]_{\triangle}\right]_{j}=\left(S / P^{\beta}\right)_{(c j-d i+\alpha, e j-i+\beta)} .
$$

We will show that

$$
\left(S / P^{\beta}\right)_{(c j-d i+\alpha, e j-i+\beta)}=0 .
$$

The case $i=0$ will be dealt with separately. Assume $i>0$. Clearly (7) holds if $e j-i+\beta \geq \beta$, that is, if $e j \geq i$. To complete the argument for (7), it is enough to show that $c j-d i+\alpha<0$ for $e j<i$. Let $a=q c+r$; $0 \leq r<c$. Then

$$
\left\lceil\frac{a}{c}\right\rceil= \begin{cases}q+1 & \text { if } r \neq 0 \\ q & \text { if } r=0 .\end{cases}
$$


Therefore,

$$
c j-d i+\alpha= \begin{cases}c j-d i+c-r & \text { if } r \neq 0 \\ c j-d i & \text { if } r=0 .\end{cases}
$$

Assume $r=0$ and $e j<i$. It is easy to see that

$$
c j-d i<i\left(\frac{c}{e}-d\right)<i\left(\frac{2 d}{3 e}-d\right)<0 .
$$

Assume $r \neq 0$ and $e j<i$. By assumption, we have $j \leq i / e-1 / e$ and $c j-d i+c-r<c(j+1)-i d$. One has

$$
c(j+1)-d i \leq c\left(\frac{i}{e}-\frac{1}{e}+1\right)-d i=i\left(\frac{c}{e}-d\right)-\frac{c}{e}+c .
$$

As $d / 2 \leq c<2 d / 3$ and $e>0$, we may write

$$
\frac{c}{e}-d<\frac{2 d}{3 e}-d=\frac{d(2-3 e)}{3 e} \text { and }-\frac{c}{e} \leq-\frac{d}{2 e} .
$$

Thus, by (9) and (10), we have

$$
\begin{aligned}
c(j+1)-d i & <i\left(\frac{d(2-3 e)}{3 e}\right)-\frac{d}{2 e}+\frac{2 d}{3} \\
& =\frac{d}{6 e}[i(4-6 e)+(4 e-3)] .
\end{aligned}
$$

It is easy to see that $i(4-6 e)+(4 e-3)<0$ for all $i>0$. Assume $i=0$. Denote by $\mathbf{C}$ the complex $(\mathbf{F} \otimes R(\alpha, \beta))_{\triangle}$. If $H_{0}(\mathbf{C}) \neq 0$, then either $e j+\beta<\beta$ or $c j+\alpha \geq 0$ in (7). Thus, $H_{0}(\mathbf{C}) \neq 0$ if $-\alpha / c \leq j<0$, which is impossible since $j$ has to be an integer and, by previous discussion in (8), one has $-1<-\alpha / c \leq 0$. Since $H_{i}(\mathbf{C})=0$ for all $i \geq 0$, we have the following exact complex $\mathbf{C}$ :

$$
\begin{aligned}
0 \longrightarrow R(\alpha,-i+1)_{\triangle} \longrightarrow \cdots \longrightarrow & R(\alpha,-1)_{\triangle} \\
& \longrightarrow R(\alpha, 0)_{\triangle} \longrightarrow R(\alpha, \beta)_{\triangle} \longrightarrow 0 .
\end{aligned}
$$

From the exact complex $\mathbf{C}$, we build another complex:

$$
\mathbf{T}: 0 \longrightarrow R(\alpha,-i+1)_{\triangle} \longrightarrow \cdots \longrightarrow R(\alpha,-1)_{\triangle} \longrightarrow R(\alpha, 0)_{\triangle} \longrightarrow 0 .
$$


Then the homology of the new complex $\mathbf{T}$ is given by

$$
H_{i}(\mathbf{T})= \begin{cases}R(\alpha, \beta) \triangle & \text { if } i=0 ; \\ 0 & \text { if } i>0 .\end{cases}
$$

Thus, applying Lemma 2.7 to the complex $\mathbf{T}$, one has

$$
\operatorname{reg}_{R_{\triangle}} R(\alpha, \beta)_{\triangle} \leq \max \left\{\operatorname{reg}_{R_{\triangle}} R(\alpha,-i)_{\triangle}-i: i \geq 0\right\} .
$$

Note that, by Case 1 , we have $\operatorname{reg}_{R \triangle} R(\alpha,-i)_{\triangle} \leq\lceil i / e\rceil$, since $\lceil-\alpha / c\rceil \leq$ $\lceil i / e\rceil$. Thus, we conclude that $\operatorname{reg}_{R_{\triangle}} R(\alpha, \beta)_{\triangle} \leq 0$. Hence, Claim (b) follows.

Remark 2.11. Note that Proposition 2.10 is an extension of [9, Theorem 6.2] for certain bigraded complete intersection ideal. Note also that the statement of Proposition 2.10 is similar to (and more general than) [8, Proposition 2.2].

3. Improvement of bounds. Let $S=K\left[x_{1}, \ldots, x_{n}\right]$ be a polynomial ring. Let $I$ be a homogeneous ideal in $S$ generated by a regular sequence $f_{1}, f_{2}, \ldots, f_{k}$, of homogeneous forms of degree $d$. Let $c$ and $e$ be positive integers. Consider the $K$-subalgebra of $S$ generated by the homogeneous forms of degree $e d+c$ in the ideal $I^{e}$, that is, $K\left[\left(I^{e}\right)_{e d+c}\right]$. We have seen that $K\left[\left(I^{e}\right)_{e d+c}\right]$ is the $(c, e)$-diagonal subalgebra of Rees $(I)$.

By [9, Corollary 6.10], $K\left[\left(I^{e}\right)_{e d+c}\right]$ is shown to be quadratic if $c \geq d / 2$ and Koszul if $c \geq d(k-1) / k$. In [9, page 900], the authors mentioned that they expect $K\left[\left(I^{e}\right)_{e d+c}\right]$ to be Koszul also for $d / 2 \leq c<d(k-1) / k$. For $k=1,2$, it is obvious that $K\left[\left(I^{e}\right)_{e d+c}\right]$ is Koszul. The very first nontrivial instance of this problem occurs for $d=2$ and $k=3$, in which case the only possible value is $c=1$. For $c=1$, the answer is positive and solved by Caviglia and Conca [8].

In this Section, for $k=3$ and for any $d$, we prove that $K\left[\left(I^{e}\right)_{e d+c}\right]$ is Koszul as soon as $c \geq d / 2$ and $e>0$. The main theorem of this section is as follows.

Theorem 3.1. Let $I$ be an ideal of the polynomial ring $K\left[x_{1}, \ldots, x_{n}\right]$ generated by a regular sequence $f_{1}, f_{2}, f_{3}$, of homogeneous forms of degree $d$. Then $K\left[\left(I^{e}\right)_{e d+c}\right]$ is Koszul for all $c \geq d / 2$ and $e>0$. 
We consider the Rees algebra, Rees $(I) \subset S[t]$ of $I$ with its standard bigraded structure induced by $\operatorname{deg}\left(x_{i}\right)=(1,0)$ and $\operatorname{deg}\left(f_{j} t\right)=$ $(0,1)$. It can be realized as a quotient of the polynomial ring $S^{\prime}=$ $K\left[x_{1}, \ldots, x_{n}, t_{1}, t_{2}, t_{3}\right]$ bigraded with $\operatorname{deg}\left(x_{i}\right)=(1,0)$ and $\operatorname{deg}\left(t_{j}\right)=$ $(0,1)$, by the ideal $J$ generated by the 2 -minors of

$$
M=\left(\begin{array}{ccc}
f_{1} & f_{2} & f_{3} \\
t_{1} & t_{2} & t_{3}
\end{array}\right) .
$$

Let $h_{1}, h_{2}$ and $h_{3}$ be the 2-minors of $M$ with the appropriate sign, say $h_{i}$ equal to $(-1)^{i+1}$ times the minor of $M$ obtained by deleting the $i$ th column. Hence,

$$
J=I_{2}(M)=\left(h_{1}, h_{2}, h_{3}\right) .
$$

The sign convention is chosen so that the rows of the matrix $M$ are syzygies of $h_{1}, h_{2}$ and $h_{3}$. We will use the following lemma to prove Theorem 3.1.

Lemma 3.2. [8, Lemma 3.1] (Technical lemma).

(i) $h_{1}, h_{2}$ form a regular $S^{\prime}$-sequence.

(ii) $\left(h_{1}, h_{2}\right): h_{3}=\left(f_{3}, t_{3}\right)$.

(iii) $\left(h_{1}, h_{2}\right): t_{3}=J$.

(iv) $\left(t_{3}, h_{1}, h_{2}\right): f_{3}=\left(t_{1}, t_{2}, t_{3}\right)$.

Remark 3.3. Note that Lemma 3.2 is proved for $d=2$ in [8, Lemma 3.1]. We observe that the proof of [8, Lemma 3.1] is independent of the degrees of polynomial. Hence, Lemma 3.2 also holds for all $d$.

We are now ready for the proof of Theorem 3.1:

Proof. Recall that $K\left[\left(I^{e}\right)_{e d+c}\right]$ is Koszul for all $c \geq 2 d / 3$ and $e>0$ [9, Corollary 6.10]. We will show that $K\left[\left(I^{e}\right)_{e d+c}\right]$ is Koszul also for $d / 2 \leq c<2 d / 3$ and $e>0$.

We set $B=S^{\prime} /\left(h_{1}, h_{2}\right)$. By Lemma 3.2 (i), we may apply to $B$ the result of Proposition 2.10. Hence, $B_{\triangle}$ is Koszul. One also has $B / h_{3} B=\operatorname{Rees}(I)$. It is enough to show for the $(c, e)$-diagonal subalgebra of Rees $(I)$, one has

$$
\operatorname{reg}_{B_{\triangle}}\left(\operatorname{Rees}(I)_{\triangle}\right) \leq 1 .
$$


Since $h_{3} t_{3}=0$ in $B$, we have a complex

$$
\begin{aligned}
\mathbf{F}: \cdots \stackrel{t_{3}}{\longrightarrow} B(-2 d,-3) \stackrel{h_{3}}{\longrightarrow} B(-d,-2) \stackrel{t_{3}}{\longrightarrow} B(-d,-1) \\
\stackrel{h_{3}}{\longrightarrow} B \longrightarrow 0
\end{aligned}
$$

where $F_{0}=B, F_{2 i}=B(-i d,-2 i)$ and $F_{2 i+1}=B(-(i+1) d,-2 i-1)$. The homology of $\mathbf{F}$ can be described by using Lemma 3.2

$$
H_{k}(\mathbf{F})= \begin{cases}\operatorname{Rees}(I) & \text { if } k=0, \\ 0 & \text { if } k=2 i \text { and } i>0, \\ {\left[S^{\prime} /\left(t_{1}, t_{2}, t_{3}\right)\right](-(i+1) d-d,-2 i-1)} & \text { if } k=2 i+1 \text { and } i \geq 0 .\end{cases}
$$

The assertion for $k=0$ holds by construction. For $k$ even and positive, it holds because of Lemma 3.2. For $k$ odd and positive by Lemma 3.2 (ii), we have

$$
H_{2 i+1}(\mathbf{F})=\frac{\left(t_{3}, f_{3}\right)}{\left(t_{3}, h_{1}, h_{2}\right)}(-(i+1) d,-2 i-1) .
$$

Hence, $H_{2 i+1}(\mathbf{F})$ is cyclic generated by the residue class of $f_{3} \bmod$ $\left(t_{3}, h_{1}, h_{2}\right)$ that has degree $(-(i+1) d-d,-2 i-1)$. Using Lemma 3.2 (iv) and keeping track of the degree, we get the desired result. Applying the $-\triangle$ functor to (11), we obtain a complex $\mathbf{F}_{\triangle}$ :

$$
\begin{aligned}
& \cdots \longrightarrow B(-2 d,-3)_{\triangle} \longrightarrow B(-d,-2)_{\triangle} \\
& \longrightarrow B(-d,-1)_{\triangle} \longrightarrow B_{\triangle} \longrightarrow 0
\end{aligned}
$$

where

$$
\left(F_{k}\right)_{\triangle}= \begin{cases}B_{\triangle} & \text { if } k=0 \\ B(-i d,-2 i) \triangle & \text { if } k=2 i \\ B(-(i+1) d,-2 i-1) \triangle & \text { if } k=2 i+1 .\end{cases}
$$

Note that $H_{2 i}\left(\mathbf{F}_{\triangle}\right)=0$ and $H_{0}\left(\mathbf{F}_{\triangle}\right)=\operatorname{Rees}(I)_{\triangle}$. We observe that $H_{2 i+1}\left(\mathbf{F}_{\triangle}\right)$ is not necessarily zero for all $e \geq 1$. Assume $e \geq 2$. Then we claim that $H_{2 i+1}\left(\mathbf{F}_{\triangle}\right)=0$. Take the $j$-th degree component

$$
\left(H_{2 i+1}(\mathbf{F})_{\triangle}\right)_{j}=\left[S^{\prime} /\left(t_{1}, t_{2}, t_{3}\right)\right]_{(-(i+1) d-d+j c,-2 i-1+j e)} .
$$

We will show that

$$
\left[S^{\prime} /\left(t_{1}, t_{2}, t_{3}\right)\right]_{(-(i+1) d-d+j c,-2 i-1+j e)}=0 .
$$


Clearly, (13) holds if $-2 i-1+e j \geq 1$, that is, if $e j \geq 2(i+1)$. So, it is enough to show that $-(i+1) d-\bar{d}+j c<0$ for $e j<2(i+1)$, that is, $j<(i+2) d / c$ for $j<2(i+1) / e$. This is an easy consequence of the following inequalities:

$$
\frac{2(i+1)}{e}<\frac{3(i+2)}{2}<\frac{(i+2) d}{c} .
$$

Assume $e=1$. We know that $H_{2 i+1}\left(\mathbf{F}_{\triangle}\right)=H_{2 i+1}(\mathbf{F})_{\triangle}$. Take the $j$-th degree component of $H_{2 i+1}(\mathbf{F})_{\triangle}$. Then $\left(H_{2 i+1}(\mathbf{F})_{\triangle}\right)_{j}=0$ if $-(2 i+1)+j \geq 1$, that is, if $j \geq 2 i+2$. So, the largest degree of a non zero component of $H_{2 i+1}\left(\mathbf{F}_{\triangle}\right)$ is at most $2 i+1$. Therefore, by (1), one has $\operatorname{reg}_{B \triangle} H_{k}\left(\mathbf{F}_{\triangle}\right) \leq \operatorname{reg}_{S^{\prime}} H_{k}\left(\mathbf{F}_{\triangle}\right) \leq k$ for all $k \geq 1$. Applying Lemma 2.7 to (12), we obtain

$$
\operatorname{reg}_{B_{\triangle}}\left(\operatorname{Rees}(I)_{\triangle}\right) \leq \sup \left\{\alpha^{\prime}, \beta^{\prime}\right\},
$$

where

$$
\alpha^{\prime}=\sup \left\{\operatorname{reg}_{B_{\triangle}}\left(F_{k}\right)_{\triangle}-k: k \geq 0\right\}
$$

and

$$
\beta^{\prime}=\sup \left\{\operatorname{reg}_{B_{\triangle}} H_{k}\left(\mathbf{F}_{\triangle}\right)-(k+1): k \geq 1\right\} .
$$

Since $B$ is defined by a regular sequence of elements of bidegree $(d, 1)$, we may apply Proposition 2.10 to (12):

$$
\operatorname{reg}_{B_{\triangle}}\left(F_{k}\right)_{\triangle} \leq \begin{cases}\max \left\{\left\lceil\frac{i d}{c}\right\rceil,\left\lceil\frac{2 i}{e}\right\rceil\right\} & \text { if } k=2 i, \\ \max \left\{\left\lceil\frac{(i+1) d}{c}\right\rceil,\left\lceil\frac{2 i+1}{e}\right\rceil\right\} & \text { if } k=2 i+1\end{cases}
$$

Since $3 / 2<d / c \leq 2$, we conclude that $\alpha^{\prime} \leq 1$. Since $\operatorname{reg}_{B \triangle} H_{k}\left(\mathbf{F}_{\triangle}\right) \leq k$ for all $k \geq 1$, we conclude that $\beta^{\prime} \leq-1$. Therefore, by (14), one has

$$
\operatorname{reg}_{B_{\triangle}}\left(\operatorname{Rees}(I)_{\triangle}\right) \leq 1 \text {. }
$$

Thus, we conclude that Rees $(I)_{\triangle}$ is Koszul.

Remark 3.4. We observe that, in the proof of [8, Theorem 3.2], $H_{k}(\mathbf{F})_{\triangle}=0$ for all $k$, whereas in our case this is true for all $e \geq 2$, and not for $e=1$. This affects the proof of Theorem 3.1 much more than that of [8, Theorem 3.2]. To achieve our goal, we first have to deduce Lemma 2.7. We use the fact that, if the homology module is non zero and its regularity is bounded by the homology module at zero position, then by (1), Lemma 2.7 and Proposition 2.10, we conclude the proof. 
4. More general base rings. In this section, the two main results that we generalize are [9, Theorem 6.2] and [9, Corollary 6.10]. We show that the Koszulness property holds even if the assumption of the polynomial ring is replaced by a Koszul ring.

Conca et al. in [9, page 900] posed two interesting questions, one of which was positively answered by Aramova, Crona and De Negri [1] who showed that for an arbitrary bigraded standard algebra $R$, the defining ideal of $R_{\triangle}$ has quadratic Grobner basis for $c, e \gg 0$, and another one by Blum [5], who showed that all the diagonal algebras of bigraded standard Koszul algebra $R$ are Koszul. We will use these results in the proofs of Theorem 4.1 and Theorem 4.5.

Let $A$ and $B$ be two standard graded Koszul algebras. Let $A=$ $K\left[A_{1}\right]$, where $A_{1}=\left\langle X_{1}, \ldots X_{m}\right\rangle$ is a $K$ vector space generated by linear forms with $\operatorname{deg}\left(X_{i}\right)=1$. Similarly, let $B=K\left[B_{1}\right]$, where $B_{1}=\left\langle Y_{1}, \ldots Y_{n}\right\rangle$ is a $K$ vector space generated by linear forms with $\operatorname{deg}\left(Y_{j}\right)=1$. We set $T=A \otimes_{K} B$. Then $T$ is bigraded standard by setting $\operatorname{deg}\left(X_{i}\right)=(1,0)$ and $\operatorname{deg}\left(Y_{j}\right)=(0,1)$. Let $R$ be a bigraded quotient of $T$, that is, $R=T / I$ for some bihomogeneous ideal $I$ of $T$.

Let $c$ and $e$ be positive integers. We will study the Koszul property of $(c, e)$-diagonal subalgebra $R_{\triangle}$ of bigraded algebra $R$. Consider the bigraded free resolution of $R$ over $T$ :

$$
\cdots \longrightarrow F_{i} \longrightarrow \cdots \longrightarrow F_{1} \longrightarrow T \longrightarrow R \longrightarrow 0,
$$

where

$$
F_{i}=\bigoplus_{(a, b) \in \mathbf{N}^{2}} T(-a,-b)^{\beta_{i,(a, b)}}
$$

Set

$$
t_{i, 1}=\max \left\{a: \text { there exists } b \text { such that } \beta_{i,(a, b)} \neq 0\right\},
$$

and

$$
t_{i, 2}=\max \left\{b: \text { there exists } a \text { such that } \beta_{i,(a, b)} \neq 0\right\} .
$$

With this notation, the following is a generalization of $[\mathbf{9}$, Theorem $6.2]$.

Theorem 4.1. Let $A$ and $B$ be standard graded Koszul algebras. We set $T=A \otimes_{K} B$ and $R$ a bi-graded quotient of $T$. Then: 
(i) $T$ is Koszul and $\operatorname{reg}_{T} R$ is finite.

(ii) If $c \geq \sup \left\{t_{i, 1} /(i+1): i \geq 1\right\} \in \mathbf{R}$ and $e \geq \sup \left\{t_{i, 2} /(i+1)\right.$ : $i \geq 1\} \in \mathbf{R}$, then $R_{\triangle}$ is Koszul.

(iii) In particular, if $c \geq\left(\operatorname{reg}_{T} R-1\right) / 2$ and $e \geq\left(\operatorname{reg}_{T} R-1\right) / 2$, then $R_{\triangle}$ is Koszul.

Proof. For (i), $T$ is Koszul by [4] and $\operatorname{reg}_{T} R$ is finite by [3, Theorem 1]. For (ii), applying $-\triangle$ functor to the bigraded free resolution (15) of $R$ over $T$, one obtains an exact complex:

$$
\cdots \longrightarrow\left(F_{i}\right)_{\triangle} \longrightarrow \cdots \longrightarrow\left(F_{1}\right)_{\triangle} \longrightarrow T_{\triangle} \longrightarrow R_{\triangle} \longrightarrow 0,
$$

of $T_{\triangle \text {-modules, where }}$

$$
\left(F_{i}\right)_{\triangle}=\bigoplus_{(a, b) \in \mathbf{N}^{2}} T(-a,-b)_{\triangle}^{\beta_{i,(a, b)}} .
$$

Note that $T_{\triangle}=A^{(c)} \otimes B^{(e)}$, where $A^{(c)}$ denotes the $c$-th Veronese subring of $A$, and $B^{(e)}$ denotes the $e$-th Veronese subring of $B$. Note that $T_{\triangle}$ is Koszul [5, Theorem 2.1]. To show that $R_{\triangle}$ is Koszul, it is enough to show that

$$
\operatorname{reg}_{T_{\triangle}} R_{\triangle} \leq 1
$$

Applying [9, Lemma 6.3 (ii)] to (17), we get

$$
\operatorname{reg}_{T_{\triangle}} R_{\triangle} \leq \sup \left\{\operatorname{reg}_{T_{\triangle}}\left(F_{i}\right)_{\triangle}-i: i \geq 1\right\} .
$$

Thus, it is enough to show that

$$
\operatorname{reg}_{T_{\triangle}}\left(F_{i}\right)_{\triangle}-i \leq 1 \text { for all } i \geq 1 .
$$

Since

$$
\left(F_{i}\right)_{\triangle}=\bigoplus_{(a, b) \in \mathbf{N}^{2}} T(-a,-b)_{\triangle}^{\beta_{i,(a, b)}},
$$

one has

$$
\operatorname{reg}_{T_{\triangle}}\left(F_{i}\right)_{\triangle}=\max \left\{\operatorname{reg}_{T_{\triangle}} T(-a,-b) \triangle: \beta_{i,(a, b)} \neq 0\right\} .
$$

Now we need to evaluate $\operatorname{reg}_{T_{\triangle}} T(-a,-b)_{\triangle}$. We denote by $V_{A}(c, \alpha)$, the Veronese modules of $A$, that is, $V_{A}(c, \alpha)=\bigoplus_{s \in \mathbf{N}} A_{s c+\alpha}$ for $\alpha=0, \ldots, c-1$. Similarly, denote $V_{B}(e, \beta)$, the Veronese modules 
of $B$. Hence, for the shifted module $T(-a,-b)_{\triangle}$ we can write

$$
\begin{aligned}
T(-a,-b)_{\triangle} & =\bigoplus_{s}\left[A_{s c-a} \otimes B_{s e-b}\right] \\
& =V_{A}(c, \alpha)\left(-\left\lceil\frac{a}{c}\right\rceil\right) \underline{\otimes} V_{B}(e, \beta)\left(-\left\lceil\frac{b}{e}\right\rceil\right),
\end{aligned}
$$

where $\alpha=-a \bmod (c), 0 \leq \alpha \leq c-1$ and $\beta=-b \bmod (e)$, $0 \leq \beta \leq e-1$.

The Veronese modules $V_{A}(c, \alpha)$ and $V_{B}(e, \beta)$ have a linear resolutions as an $A^{(c)}$-module and $B^{(c)}$-module, respectively, see [6, Lemma 5.1]. Hence, by (2), one has

$$
\operatorname{reg}_{T \Delta} T(-a,-b)_{\triangle}=\max \left\{\left\lceil\frac{a}{c}\right\rceil,\left\lceil\frac{b}{e}\right\rceil\right\} .
$$

Thus, by (16), (18), (19) and (20), we conclude that $R_{\triangle}$ is Koszul, provided that

$$
\max \left\{\left\lceil\frac{t_{i, 1}}{c}\right\rceil,\left\lceil\frac{t_{i, 2}}{e}\right\rceil\right\} \leq i+1 \text { for all } i \geq 1 .
$$

From (21), we conclude that if $c \geq \sup \left\{t_{i, 1} /(i+1): i \geq 1\right\}$ and $e \geq \sup \left\{t_{i, 2} /(i+1): i \geq 1\right\}$, then $R_{\triangle}$ is Koszul. By definition, one has $t_{i, 1} \leq t_{i} \leq \operatorname{reg}_{T} R-i$. Thus, we have:

$$
\frac{t_{i, 1}}{i+1} \leq \frac{t_{i}}{i+1} \leq \frac{\operatorname{reg}_{T} R-i}{i+1} .
$$

We know that $\operatorname{reg}_{T} R$ is finite. Notice that $\frac{\operatorname{reg}_{T} R-i}{i+1}$ is a decreasing function of $i$, as $i$ varies over the natural numbers. Taking the sup in (22), we get

$$
\sup \left\{\frac{t_{i, 1}}{i+1}: i \geq 1\right\} \leq \sup \left\{\frac{t_{i}}{i+1}: i \geq 1\right\} \leq \sup \left\{\frac{\operatorname{reg}_{T} R-i}{i+1}: i \geq 1\right\} .
$$

Similarly, the other case

$$
\sup \left\{\frac{t_{i, 2}}{i+1}: i \geq 1\right\} \leq \sup \left\{\frac{t_{i}}{i+1}: i \geq 1\right\} \leq \sup \left\{\frac{\operatorname{reg}_{T} R-i}{i+1}: i \geq 1\right\} .
$$

Note that

$$
\sup \left\{\frac{\operatorname{reg}_{T} R-i}{i+1}: i \geq 1\right\} \leq \frac{\operatorname{reg}_{T} R-1}{2} .
$$


Thus, we observe that the numbers $\sup \left\{t_{i, 1} /(i+1): i \geq 1\right\}$ and $\sup \left\{t_{i, 2} /(i+1): i \geq 1\right\}$ are in fact some finite real numbers, bounded by $\left(\operatorname{reg}_{T} R-1\right) / 2$. Thus, claims (ii) and (iii) follow.

Remark 4.2. Note that in the Theorem 4.1, if we take $A=$ $K\left[x_{1}, \ldots, x_{m}\right]$ and $B=K\left[y_{1}, \ldots, y_{n}\right]$, then we will re-obtain [9, Theorem 6.2].

The following is the generalization of [9, Corollary 6.9].

Corollary 4.3. Let I be the homogeneous ideal of a standard graded Koszul ring A. Let d denote the highest degree of a minimal generator of $I$. Then there exist integers $c_{0}, e_{0}$ such that the $K$-algebra $K\left[\left(I^{e}\right)_{e d+c}\right]$ is Koszul for all $c \geq c_{0}$ and $e \geq e_{0}$.

Proof. Let $B=K\left[t_{1}, \ldots, t_{k}\right]$ in Theorem 4.1. Then $T=A\left[t_{1}, \ldots, t_{k}\right]$ is a polynomial extension of $A$. Note that $T$ is a bigraded standard algebra by setting $\operatorname{deg} X_{i}=(1,0)$ and $\operatorname{deg} t_{j}=(0,1)$. By replacing $I$ with the ideal generated by $I_{d}$, we may assume that $I$ is generated by forms of degree $d$. Then Rees $(I) \subset A[t]$ is a bigraded standard algebra by setting $\operatorname{deg} X_{i}=(1,0)$ and $\operatorname{deg} f t=(0,1)$ for all $f \in I_{d}$. Moreover, Rees $(I)$ can also be realized as the bigraded quotient of $T$.

By Theorem 4.1 (i), we have $T$ is Koszul and $\operatorname{reg}_{T} \operatorname{Rees}_{(I)}$ is finite. Note that the numbers $c$ and $e$ exist from Theorem 4.1 (ii) such that Rees $(I)_{\triangle}$ is Koszul. In particular, if $c, e \geq\left(\operatorname{reg}_{T} \operatorname{Rees}(I)-1\right) / 2$, then Rees $(I)_{\triangle}$ is Koszul by Theorem 4.1 (iii). Thus, the claim follows, since Rees $(I)_{\triangle}=K\left[\left(I^{e}\right)_{e d+c}\right]$.

Remark 4.4. In Corollary 4.3, the integers $c_{0}$ and $e_{0}$ can be computed explicitly whenever one knows the shifts in the bigraded free resolution of Rees $(I)$ over the Koszul ring $T$. For instance, when $I$ is a complete intersection ideal generated by homogeneous forms of degree $d$, one has Theorem 4.5.

The following is a generalization of [9, Corollary 6.10].

Theorem 4.5. Let $A$ be a standard graded Koszul ring. Let I be an ideal of A generated by a regular sequence $f_{1}, f_{2}, \ldots, f_{k}$, of homogeneous 
forms of degree $d$. Then $K\left[\left(I^{e}\right)_{e d+c}\right]$ is Koszul for all $c \geq d(k-1) / k$ and $e>0$.

Proof. Let $A=K\left[A_{1}\right]$, where $A_{1}=\left\langle X_{1}, \ldots X_{m}\right\rangle$ is a $K$ vector space generated by linear forms with $\operatorname{deg}\left(X_{i}\right)=1$. Let $A^{\prime}=A\left[t_{1}, \ldots, t_{k}\right]$ be a polynomial extension of $A$. Then $A^{\prime}$ is a bigraded standard algebra by setting $\operatorname{deg} X_{i}=(1,0)$ and $\operatorname{deg} t_{j}=(0,1)$. Let $I$ be an ideal of $A$ generated by a regular sequence $f_{1}, f_{2}, \ldots, f_{k}$, of homogeneous forms of degree $d$. Then Rees $(I) \subset A[t]$ is a bigraded standard algebra by setting $\operatorname{deg} X_{i}=(1,0)$ and $\operatorname{deg} f_{j} t=(0,1)$. Let

$$
\phi: A\left[t_{1}, t_{2}, \ldots, t_{k}\right] \longmapsto A[I t]
$$

be the surjective map by sending $t_{j}$ to $f_{j} t$. Since $I$ is a complete intersection ideal, one has

$$
\operatorname{ker}(\phi)=I_{2}\left(\begin{array}{cccc}
f_{1} & f_{2} & \cdots & f_{k} \\
t_{1} & t_{2} & \cdots & t_{k}
\end{array}\right) .
$$

The resolution of $A[I t]$ over $A^{\prime}$ is given by the Eagon-Northcott complex (23). We know that $\operatorname{ker}(\phi)$ is a determinantal ideal and grade $(\operatorname{ker}(\phi))=$ $k-1$; hence, the Eagon-Northcott complex (23) is the minimal free resolution of $A[I t]$ over $A^{\prime}$ :

$$
0 \longrightarrow F_{k-1} \longrightarrow \cdots \longrightarrow F_{1} \longrightarrow F_{0} \longrightarrow A[I t] \longrightarrow 0,
$$

where

$$
F_{i}=\bigoplus_{j=1}^{i} A^{\prime}(-j d,-i-1+j)^{\sharp_{i}} .
$$

Here $\sharp_{i}$ denotes some integer which is irrelevant in our discussion. Applying the $-\triangle$ functor to (23), one obtains an exact complex:

$$
0 \longrightarrow\left(F_{k-1}\right)_{\triangle} \longrightarrow \cdots \longrightarrow\left(F_{1}\right)_{\triangle} \longrightarrow\left(F_{0}\right)_{\triangle} \longrightarrow A[I t]_{\triangle} \longrightarrow 0
$$

of $A_{\triangle}^{\prime}$-modules, where

$$
\left(F_{i}\right)_{\triangle}=\bigoplus_{j=1}^{i} A^{\prime}(-j d,-i-1+j)_{\triangle}^{\sharp i} .
$$

From the exact complex (24), we build another complex:

$$
\mathbf{F}: 0 \longrightarrow\left(F_{k-1}\right)_{\triangle} \longrightarrow \cdots \longrightarrow\left(F_{1}\right)_{\triangle} \longrightarrow\left(F_{0}\right)_{\triangle} \longrightarrow 0
$$


of $A_{\triangle}^{\prime}$-modules. Then the homology of the new complex $\mathbf{F}$ is given by

$$
H_{i}(\mathbf{F})= \begin{cases}A[I t] \triangle & \text { if } i=0 \\ 0 & \text { if } i>0\end{cases}
$$

Thus, applying Lemma 2.7 to the complex $\mathbf{F}$, one has

$$
\operatorname{reg}_{A_{\triangle}^{\prime}}\left(A[I t]_{\triangle}\right) \leq \sup \left\{\operatorname{reg}_{A_{\triangle}^{\prime}}\left(F_{i}\right)_{\triangle}-i: i=1,2, \ldots, k-1\right\} .
$$

Note that $A_{\triangle}^{\prime}$ is Koszul [5, Theorem 2.1]. To show that $A[I t]_{\triangle}$ is Koszul, it is enough to show that

$$
\operatorname{reg}_{A_{\triangle}^{\prime}}\left(F_{i}\right)_{\triangle}-i \leq 1 \text { for all } i=1,2, \ldots, k-1 .
$$

One has

$$
\operatorname{reg}_{A_{\triangle}^{\prime}}\left(F_{i}\right)_{\triangle}=\max \left\{\operatorname{reg}_{A_{\triangle}^{\prime}} A^{\prime}(-j d,-i-1+j) \triangle: j=1,2, \ldots, i\right\} .
$$

By a similar argument as used in Theorem 4.1 to obtain equation (20), we get

$$
\operatorname{reg}_{A_{\triangle}^{\prime}}\left(F_{i}\right)_{\triangle}=\max \left\{\left\lceil\frac{j d}{c}\right\rceil,\left\lceil\frac{i+1-j}{e}\right\rceil: j=1,2, \ldots, i\right\} .
$$

Thus, we have

$$
\operatorname{reg}_{A_{\triangle}^{\prime}}\left(F_{i}\right)_{\triangle}=\max \left\{\left\lceil\frac{i d}{c}\right\rceil,\left\lceil\frac{i}{e}\right\rceil\right\} .
$$

Therefore, by (25) and (26), we conclude that $K\left[\left(I^{e}\right)_{e d+c}\right]$ is Koszul if $c \geq d(k-1) / k$ and $e>0$.

We conclude the section with one final remark, and with an open question.

Remark 4.6. The claim by Conca, et al. in [9, page 900] together with Theorems 3.1 and 4.5 in this paper suggest that the following question may have a positive answer.

Question 4.7. Let $I$ be an ideal of a Koszul ring $A$ generated by a regular sequence $f_{1}, f_{2}, \ldots, f_{k}$, of homogeneous forms of degree $d$. Is it true that $K\left[\left(I^{e}\right)_{e d+c}\right]$ is Koszul for all $c \geq d / 2$ and $e>0$ ? 
Acknowledgments. The author is very grateful to Professor Aldo Conca for several useful discussions on the subject of this article. The author thanks Professor Giulio Caviglia for his helpful suggestions concerning the presentation of this article. The author is also grateful to the referee for a number of helpful suggestions for improvement of the article.

\section{REFERENCES}

1. A. Aramova, K. Crona and E. De Negri, Bigeneric initial ideals, diagonal subalgebras and bigraded Hilbert functions, J. Pure Appl. Alg. 150 (2000), 215-235.

2. A. Aramova, S. Bărcănescu and J. Herzog, On the rate of relative Veronese submodules, Rev. Roum. Math. Pures Appl. 40 (1995), 243-251.

3. L.L. Avramov and D. Eisenbud, Regularity of modules over a Koszul algebras, J. Algebra 153 (1992), 85-90.

4. J. Backelin and R. Fröberg, Koszul algebras, Veronese subrings, and rings with linear resolution, Rev. Roum. Math. Pures Appl. 30 (1985), 549-565.

5. S. Blum, Subalgebras of a bigraded Koszul algebra, J. Alg. 242, (2001), 795809.

6. W. Bruns, A. Conca and T. Römer, Koszul homology and syzygies of Veronese subalgebras, Math. Ann. 351 (2011), 761-779.

7. G. Caviglia, The pinched Veronese is Koszul, J. Alge. Combin. 30 (2009), 539-548.

8. G. Caviglia and A. Conca, Koszul property of projections of the Veronese cubic surface, Adv. Math. 234 (2013), 404-413.

9. A. Conca, J. Herzog, N.V. Trung and G. Valla, Diagonal subalgebras of bigraded algebras and embeddings of blow-ups of projective spaces, Amer. J. Math. 119 (1997), 859-901.

10. A. Conca, E. De Negri and M.E. Rossi, Koszul algebras and regularity, in Commutative algebra, Springer, New York, 2013.

11. D. Eisenbud, A. Reeves and B. Totaro, Initial ideals, Veronese subrings, and rate of algebras, Adv. Math. 109 (1994), 168-187.

12. R. Fröberg, Koszul algebras, Lect. Notes Pure Appl. Math. 205, Dekker, New York, 1999.

13. S. Goto and K. Watanabe, On graded rings I, J. Math. Soc. Japan 30 (1978), 179-213.

14. K. Kurano, E. Sato, A.K. Singh and K. Watanabe, Multigraded rings, diagonal subalgebras, and rational singularities, J. Algebra 322 (2009), 3248-3267.

15. S.B. Priddy, Koszul resolutions, Trans. Amer. Math. Soc. 152 (1970), 39-60.

16. A. Simis, N.V. Trung and G. Valla, The diagonal subalgebra of a blow-up algebra, J. Pure Appl. Alg. 125 (1998), 305-328.

Dipartimento di Matematica, Universitá di Genova, Via Dodecaneso 35, 16146 Genova, ItALY

Email address: kumar@dima.unige.it 